\title{
Evaluating Immersive Approaches to Multidimensional Information Visualization
}

\author{
Jorge A. Wagner Filho ${ }^{1}$, Carla M.D.S. Freitas ${ }^{1}$ (co-supervisor), \\ Luciana Nedel $^{1}$ (supervisor)
}

\author{
${ }^{1}$ Programa de Pós-Graduação em Computação (PPGC) \\ Instituto de Informática - Universidade Federal do Rio Grande do Sul (UFRGS)
}

$$
\text { \{jawfilho, carla, nedel\}@inf.ufrgs.br }
$$

\begin{abstract}
In this work we investigate the use of Virtual Reality for the exploration of multidimensional data represented as $3 D$ scatterplots. After an initial user study indicated that an immersive environment required less effort to find information and less navigation, but resulted in inefficient times and frequent user discomfort, we proposed and evaluated an alternative data exploration approach based on the use of physical movements, direct interaction with data at arm's reach and a virtual reproduction of the user's work desk. Through a second study, we demonstrated that this setup, named VirtualDesk, presents excellent results regarding user comfort, and performs equally or better in all tasks, amplifying data exploration while adding minimal or no time overhead.
\end{abstract}

\section{Introduction}

Three-dimensional (3D) data representations are known to offer advantages under many circumstances in the context of data visualization. For inherently spatial data, they contribute to a quicker construction of the mental model. For abstract information, they have also been demonstrated to be useful, allowing clearer spatial separation in large graphs, detection of trivariate patterns in scatterplots and more accurate projection of multidimensional data [Gracia et al. , Poco et al. 2011]. Nonetheless, their use has also been controversial, mostly because they are hindered by the occurrence of known perceptual issues: perspective distortion, foreshortening and occlusion, which make data exploration cumbersome and error-prone. Moreover, there is a relevant mismatch between $3 \mathrm{D}$ visualizations and conventional two-dimensional (2D) interaction devices, such as the mouse. Along the years, following consecutive breakthroughs in Virtual Reality (VR) research, the visualization community has progressively explored the use of immersive approaches, which combine stereoscopic displays with natural interaction as alternatives to potentially change this scenario. However, in the case of abstract information visualization, research on immersive techniques is still limited, even though advantages have already been demonstrated, for example, in graph visualization.

When providing 3D visualizations of a data set within a virtual reality environment, one usually has to provide features for supporting data analysis, and this has been

\footnotetext{
${ }^{1}$ This dissertation research was carried out in a one-year period (March 2017-March 2018), resulting in a series of peer-reviewed publications and being awarded a PPGC Honourable Mention. More information can be found at the complete text and at http://www.researchgate.net/profile/Jorge_ Wagner_Filho The work was funded by CNPq. This study was financed in part by the Coordenação de Aperfeiçoamento de Pessoal de Nível Superior - Brasil (CAPES) - Finance Code 001.
} 
recently called as Immersive Analytics [Chandler et al. 2015]. Immersive Analytics is a new research field, which combines techniques from Information Visualization, Visual Analytics, Human Computer Interaction and Virtual Reality. Due to this plethora of possible features, evaluation methods and design guidelines are even more needed.

\subsection{Research Question}

In this work, we target a specific and relevant abstract information representation: 3D dimensionally-reduced (DR) data scatterplots. Since this particular category of scatterplots, which is commonly used for multidimensional data visualization, is always analysed in terms of the distances between points, we hypothesize that it could benefit from stereoscopic displays, egocentric points of view and more natural user interfaces, characteristics that are inherent to immersive setups. Our research question is: How the use of Head-Mounted Display (HMD) based environments for the interactive visual analysis of 3D scatterplots compares to the currently used desktop-based ones?

The use of 3D scatterplots has been controversial since long before the first uses of immersive setups, with related studies dating back to the 1970s. Their application for representing dimensionally-reduced data is also often discussed in the literature. Although adding a third component could potentially reduce information loss in the dimension reduction process, results from studies on quantifying visual analysis gains have been contradictory [Poco et al. 2011, Sedlmair et al. 2013, Gracia et al.]. Few authors, however, have investigated how immersion and stereopsis may impact on these issues. Moreover, most of them have only provided preliminary results, based on technologies which have advanced enormously over the past few years [Arms et al. 1999, Raja et al. 2004]. Therefore, we contribute to the investigation of this problem by providing updated results in terms of a new interactive visualization metaphor and evaluation methodology.

\subsection{Summary of Objectives and Contributions}

Considering the context of immersive analytics applications based on 3D scatterplots obtained from DR techniques, we can state our main goal as the investigation of immersive visualization techniques that minimize errors in user perception, decrease user's workload and remain time-efficient. As a secondary goal, we focused on defining a task-based evaluation framework for these applications. In summary, our main contributions are:

1. an improved modelling of the problem of evaluating immersive visualizations of 3D dimensionally-reduced data scatterplots;

2. a task-based evaluation framework;

3. the identification of a complete set of relevant tasks for evaluation purposes;

4. a novel immersive data exploration metaphor, the VirtualDesk;

5. baseline results to be used in future work.

\section{Problem Model and Two-Step Evaluation Framework}

When exploring an immersive 3D DR data scatterplot, the performance gains attained in a task are not just a function of the difference in perceptual accuracy presented by users under different visualization conditions, but rather of its interplay with the difference in errors introduced by reducing the dimensions of a particular dataset to two or three dimensions. This new model of our problem in hand, accounting for the two different factors that influence in the final task performance outcome, is presented in Figure 1 . 


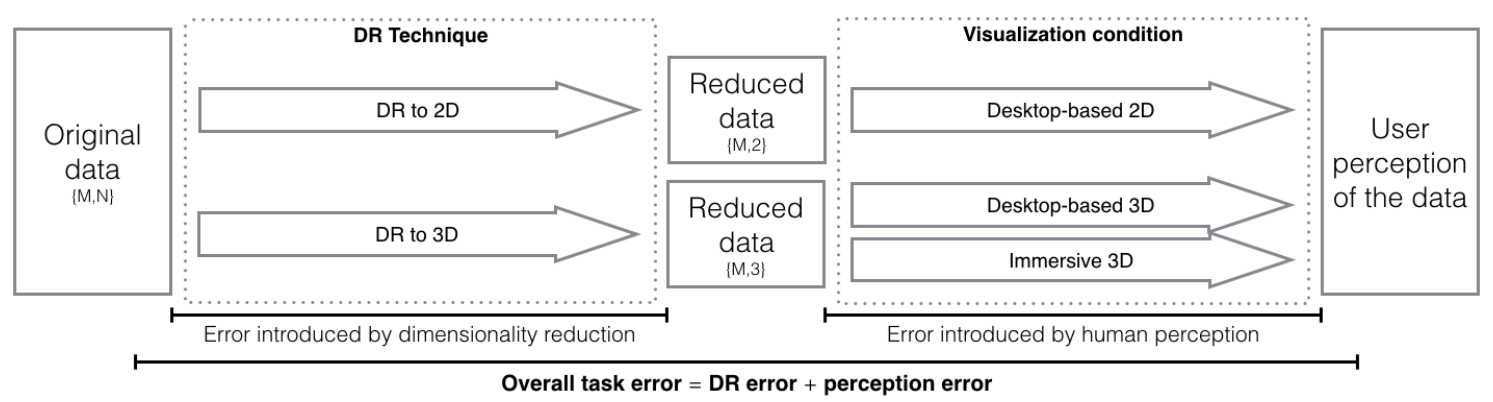

Figure 1. Visual model of our research problem. The overall task performance for each of the three scenarios will be a result of the different errors introduced: by reducing the dataset to two or three dimensions and by using a desktop-based 2D, desktop-based 3D or immersive visualization approach.

The so called DR error component is dataset-dependent, depending on the particular complexity of the data structure. This means that, for a given dataset to benefit from a 3D visualization, its content must be indeed better mapped to 3D. Moreover, the user must be able to perceive this added information appropriately, what can be challenging given the discussed issues associated with 3D representations. Based on this model, we propose an evaluation framework that aims to assess each of these variables separately. The maximum potential performance in $2 \mathrm{D}$ or $3 \mathrm{D}$ for our datasets is estimated through a task-based empirical approach. The perception and overall task errors, on the other hand, are assessed through user studies, comparing alternative visualization conditions.

For our evaluation purposes, we chose as use case the visualization of roll call voting data from the Brazilian Chamber of Deputies. This dataset is particularly interesting for visualization because the resulting spectrum is composed by more than 20 political parties, with very fuzzy ideological borders. We also consider this domain very appropriate for our goals due to the very high dimensionality of its datasets (each roll call is a dimension), its consistent application of DR techniques in the literature, and the easy definition of semantically meaningful analytical tasks. Moreover, it also appeals to different kinds of public, with potential to engage participants in exploration during our user tests.

\section{Initial Evaluation and Identification of Points for Improvement}

A first user evaluation with 30 subjects recruited on campus was conducted to compare the task performance in desktop-based (2D and 3D) and HMD-based (3D) visualization conditions, and thus evaluate the second factor in our model. In this study, a conventional gaze-directed flying navigation approach was implemented. This metaphor is meant to be simple to learn and enable an egocentric view, placing the user inside the data.

Surprisingly, however, no perception differences were observed, and similarly low errors in all conditions resulted in improvements with the addition of the third dimension with or without immersion when the dataset enabled so. Even so, on further inspection of subjective results, it was found that the HMD-based condition had required smaller effort to find information and less navigation, besides offering a much larger subjective perception of accuracy and engagement. Its main limitations, on the other hand, were the high incidence of simulator sickness, with around $40 \%$ of the participants reporting significant discomfort levels, and long task completion times. 

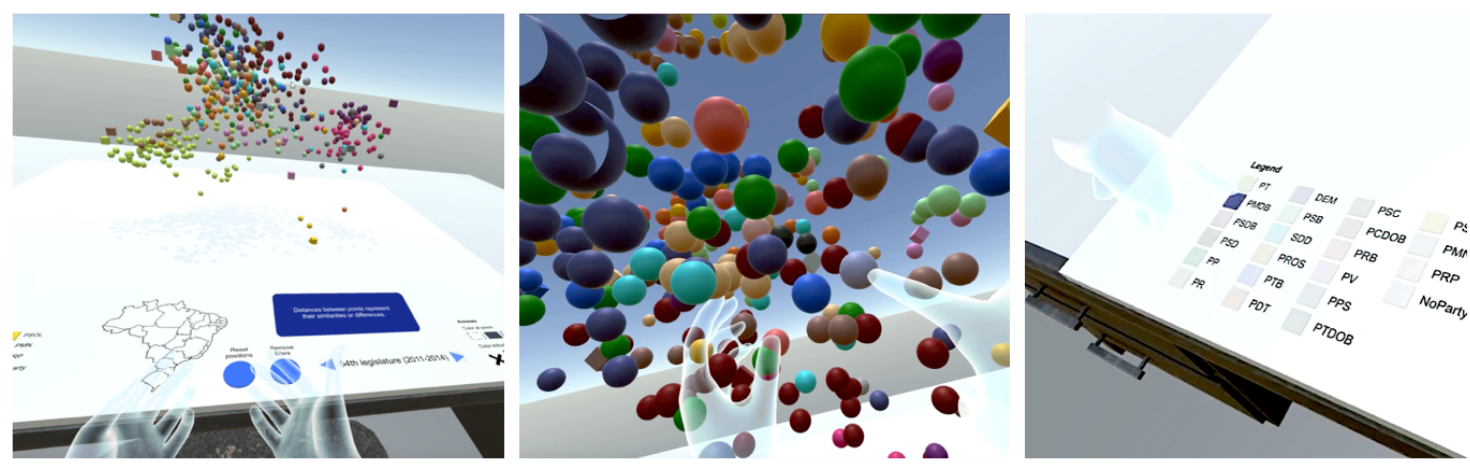

Figure 2. In the VirtualDesk metaphor, data is rendered at arm's reach and manipulated only by direct mid-air natural hand gestures. A reproduction of the user's real desk enables tangible interaction with the views and controls.

\section{VirtualDesk: A Novel Proposal for More Comfortable and Efficient Immersive Information Visualization}

In our first user study, we observed that immersive approaches may effectively aid in the exploration of multidimensional information, but that new evaluations and guidelines were still needed. Many proposed navigation approaches, for example, are impractical for actual usage. Flying metaphors, in particular, are time-consuming and often result in simulator sickness. Other approaches, such as real walking, are also unnecessarily inefficient, both in terms of time and space requirements. Moreover, how to display inherently 2D content and texts in the virtual environment is another known issue.

Aiming to circumvent these issues, we proposed and implemented an alternative data exploration approach, designed to be more fit for real usage. The metaphor, VirtualDesk, combined features from different backgrounds:

1. The dataset representation is rendered in smaller scale at arm's reach, to better benefit from proprioception, more precise body-relative interaction and stronger stereopsis and head-motion parallax [Mine et al. 1997].

2. Embodied natural data manipulation conforms with the recent concept of spatiodata coordination, i.e., a one-to-one mapping between physical and virtual actions, aiming to lower the user cognitive load [Cordeil et al. 2017].

3. A virtual desk is represented, synchronised with the user's real one. This allows tangible interaction with controls and 2D coordinated views, placed on the desk's surface [Zielasko et al. 2017].

In order to assess how the VirtualDesk prototype would perform in comparison both with conventionally used desktop-based approaches and the previously employed flying navigated immersive approach, a new study was carried out with 24 subjects. A new comparable desktop-based environment was implemented, with the same functionalities and following typical mouse and keyboard interaction approaches. Both conditions employ our visualization use case with multidimensional data projected to 3 dimensions. In order to identify the strengths and weaknesses of each, participants were asked to complete an extended set of perception and interaction tasks, inspired in previous literature.

Results showed that VirtualDesk performed equally well or better in terms of error rates across all analytical tasks, both in comparison to a standard desktop interface and to 
the previous immersive implementation with flying navigation. The added time overhead with relation to desktop was only significant in tasks with higher requirements for tabletop interaction (which required viewpoint change and also imposed certain difficulties for some users), and was generally of only a few seconds. This was despite the fact that data exploration in terms of dataset rotation was found to be 5.8 times higher. Considering that the observation from different points of view is fundamental in the comprehension of a 3D point cloud, this partially explains VirtualDesk's advantage in perception tasks.

The immersive environment also contributed to higher subjective perceptions of efficiency and engagement, while incurring minimal time overhead and generating almost no simulator sickness symptoms. Despite very similar VR exposure times in both studies, the average Simulator Sickness score in VirtualDesk was 7 times lower than in the artificially navigated version. Moreover, while in that study $40 \%$ of the users had experienced very significant discomfort levels, i.e., with scores $>=20$, now the maximum individual score was 18.3 , and $83 \%$ of the users perceived only negligible or minimal symptoms.

\section{Conclusion}

In this work, we aimed at discussing Immersive Analytics issues, by demonstrating that current off-the-shelf VR technologies may effectively aid in analytical tasks performed on abstract information visualization, even challenging previous beliefs about three-dimensional representations. We presented evaluations on a particular representation commonly for multidimensional data: 3D dimensionally-reduced data scatterplots.

The VirtualDesk data exploration metaphor proposed as part of this study is particularly promising due to its observed results for user comfort and relatively short required completion times. Considering existent implementations for immersive exploration of abstract information, most are based in the metaphors of flying through a dataset or walking around it. The former, while providing an egocentric view of the data, commonly results in simulator sickness due to the conflict with the user's real position. The latter successfully avoids this problem, but is very costly in terms of required time and space. VirtualDesk, on the other hand, achieved a very low score for simulator sickness while remaining time-efficient, and is easily integrable into a user's work environment. This makes it more convenient for real world usage, requiring only minor improvements.

\subsection{Publications}

Parts of this dissertation work have been published in the following papers. Citations are shown as indexed by Google Scholar as of May 13th, 2019.

- Jorge Alberto Wagner Filho, Marina F. Rey, Carla M.D.S. Freitas, Luciana Nedel (2017). Immersive Analytics of Dimensionally-Reduced Data Scatterplots. IEEE VIS Workshop on Immersive Analytics: Exploring Future Interaction and Visualization Technologies for Data Analytics. [2 citations]

- Jorge Alberto Wagner Filho, Marina F. Rey, Carla M.D.S. Freitas, Luciana Nedel (2018). Immersive Visualization of Abstract Information: An Evaluation on Dimensionally-Reduced Data Scatterplots. 25th IEEE Conference on Virtual Reality and 3D User Interfaces (IEEE VR 2018) [Qualis B1, 4 citations].

- Jorge Alberto Wagner Filho, Carla M.D.S. Freitas, Luciana Nedel (2018). VirtualDesk: A Comfortable and Efficient Immersive Information Visualization Approach. Computer Graphics Forum [Qualis A1, 6 citations]. 
- Jorge Alberto Wagner Filho, Carla M.D.S. Freitas, Luciana Nedel (2019). Comfortable Immersive Analytics with the VirtualDesk Metaphor: Case Studies and Perspectives. IEEE Computer Graphics and Applications [Qualis A2].

\subsection{Future Works}

Future works include the improvement of the VirtualDesk prototype based on the user study participants' feedback, and further testing under different conditions, different datasets and representations. We believe that strong candidates to benefit from this exploration metaphor would be node-link diagrams and space-time cube representations. This research is currently ongoing as part of the student's $\mathrm{PhD}$ project.

\section{References}

Arms, L., Cook, D., and Cruz-Neira, C. (1999). The benefits of statistical visualization in an immersive environment. In Proc. IEEE Virtual Reality, pages 88-95. IEEE.

Chandler, T., Cordeil, M., Czauderna, T., Dwyer, T., Glowacki, J., Goncu, C., Klapperstueck, M., Klein, K., Marriott, K., Schreiber, F., et al. (2015). Immersive analytics. In 2015 Big Data Visual Analytics (BDVA), pages 1-8. IEEE.

Cordeil, M., Bach, B., Li, Y., Wilson, E., and Dwyer, T. (2017). A design space for spatio-data coordination: Tangible interaction devices for immersive information visualisation. In Proceedings of IEEE Pacific Visualization Symposium (Pacific Vis).

Gracia, A., González, S., Robles, V., Menasalvas, E., and von Landesberger, T. New insights into the suitability of the third dimension for visualizing multivariate/multidimensional data: A study based on loss of quality quantification. $I V, 15(1)$.

Mine, M. R., Brooks Jr, F. P., and Sequin, C. H. (1997). Moving objects in space: exploiting proprioception in virtual-environment interaction. In SIGGRAPH, pages 19-26. ACM Press/Addison-Wesley Publishing Co.

Poco, J., Etemadpour, R., Paulovich, F. V., Long, T., Rosenthal, P., Oliveira, M. C. F. d., Linsen, L., and Minghim, R. (2011). A framework for exploring multidimensional data with 3d projections. Computer Graphics Forum, 30(3):1111-1120.

Raja, D., Bowman, D., Lucas, J., and North, C. (2004). Exploring the benefits of immersion in abstract information visualization. In Proc. Immersive Projection Technology Workshop, pages 61-69.

Sedlmair, M., Munzner, T., and Tory, M. (2013). Empirical guidance on scatterplot and dimension reduction technique choices. IEEE Transactions on Visualization and Computer Graphics, 19(12):2634-2643.

Zielasko, D., Weyers, B., Bellgardt, M., Pick, S., Meibner, A., Vierjahn, T., and Kuhlen, T. W. (2017). Remain seated: towards fully-immersive desktop VR. In 2017 IEEE 3rd Workshop on Everyday Virtual Reality (WEVR), pages 1-6. IEEE. 